\title{
Treatment with dietary cyclosporine has no direct effect on myocardial fibrosis and hypertension in mice
}

\begin{abstract}
The purpose of this study was to elucidate whether treatment with cyclosporine induces myocardial fibrosis and hypertension that may potentially be mediated by activation of angiotensin II (Ang II) and induction of inflammation. In C57BL/6 mice, cyclosporine at the doses of $15 \mathrm{mg} / \mathrm{kg} / \mathrm{day}$ or $30 \mathrm{mg} / \mathrm{kg} / \mathrm{day}$ was administrated by gastric gavage. In different groups, angiotensin II (Ang II) AT1 receptor blocker, telmisartan $(10 \mathrm{mg} / \mathrm{kg}$ / day) or anti-inflammatory compound, curcumin $(150 \mathrm{mg} / \mathrm{kg} /$ day $)$ was co-administered with cyclosporine. Treatment with cyclosporine for 5 weeks did not alter the protein expression, including AT1 and AT2 receptors, monocyte chemotactic protein-1, transforming growth factor $\beta 1$, collagen I and collagen III, but increased expression of angiotensin converting enzyme 2 relative to the sham control. Interstitial macrophage infiltration and myofibroblast proliferation were not changed by cyclosporine, consistent with lack of collagen deposition in the interstitial/perivascular regions. Coadministration of telmisartan or curcumin with cyclosporine also had no effect on any of the parameters measured. There was a trend in increase of blood pressure with high dose of cyclosporine alone, and decrease of blood pressure when cyclosporine was co-administered with telmisartan or curcumin, but these changes did not achieve the statistical differences among the groups. These data suggest that cyclosporine has no direct effect on myocardial fibrosis and blood pressure in healthy individuals, and it may be considered as an adjunctive therapy with commonly-used drugs to treat cardiovascular diseases.
\end{abstract}

Keywords: angiotensin II, cyclosporine, myocardial fibrosis, curcumin, telmisartan
Volume I Issue 3 - 2017

\author{
Ning-Ping Wang,' Erskine James, ${ }^{2}$ John R \\ Curcuru, 'Wei-Wei Zhang, ${ }^{3}$ Rong-Hua \\ Zheng, ${ }^{3}$ Zhi-Qing Zhao ${ }^{1,3}$ \\ 'Cardiovascular Research Laboratory, Mercer University School \\ of Medicine, USA \\ ${ }^{2}$ Department of Internal Medicine, Navicent Health, USA \\ ${ }^{3}$ Department of Physiology, Shanxi Medical University, China
}

Correspondence: Zhi-Qing Zhao, MD, PhD, Cardiovascular Research Laboratory, Department of Basic Biomedical Sciences, Mercer University School of Medicine-Savannah Campus, 1250 East 66th Street, Savannah, Georgia 3|404, USA, Tel 9|272/8208, Fax 9127218268, Email zhao_z@mercer.edu

Received: October 27, 2017 | Published: November 14, 2017

\begin{abstract}
Abbreviations: ACE2, angiotensin converting enzyme 2; Ang II, angiotensin II; MCP-1, monocyte chemotactic protein-1; TGF- $\beta 1$, transforming growth factor $\beta 1$; MAP, mean arterial pressure
\end{abstract}

\section{Introduction}

Cyclosporine, a potent immunosuppressive agent, is proved to be effective to reduce the incidence of acute organ rejection and increase early graft survival in patients who received kidney, liver, lung or heart transplant. However, the adverse effects associated with the use of cyclosporine such as nephrotoxicity and interstitial fibrosis in kidney transplant recipient have also been observed. ${ }^{1,2}$ Although the mechanisms of action related to cyclosporine mediated transplant complications are still remained to be illustrated as a matter of debate, ${ }^{3}$ previous studies have demonstrated that activation of angiotensin II (Ang II $)^{4,5}$ and production of reactive oxygen species ${ }^{6}$ might be primarily involved in the pathogenesis of chronic cyclosporine nephrotoxicity. In spontaneously hypertensive rats, a previous study has shown that cyclosporine treatment enhances angiotensin converting enzyme activity to produce Ang II, and cyclosporineinduced nephropathy can be attenuated by the Ang II AT1 receptor blocker. ${ }^{5}$ Furthermore, chronic cyclosporine nephrotoxicity could also be relieved by anti-inflammatory drugs. ${ }^{7,8}$ Under these conditions, inhibition of perivascular and interstitial fibrosis resulting from cyclosporine toxicity has been reported. In this regard, we have recently reported that the extravasation of macrophages and proliferation of myofibroblasts are the predominant features in stimulation of transforming growth factor- $\beta$ mediated fibrotic signaling in response to Ang II stimulation and oxidant production. ${ }^{9,10}$ However, it is unknown whether administration of cyclosporine has any toxic effects on the heart and blood vessels, and whether dietary cyclosporine could be selected as a conventional intervention for treatment of cardiovascular diseases. Therefore, the present study was designed to evaluate the direct effect of cyclosporine, which simulates the regimen of clinical treatment in patient, on the heart and blood pressure. To illustrate whether cyclosporine is associated with stimulation of Ang II AT1 receptor and induction of inflammation, telmisartan, the Ang II AT1 receptor blocker and curcumin, an anti-oxidant compound ${ }^{10}$ were concomitantly administered during the treatment period with dietary cyclosporine. Expression of angiotensin converting enzyme 2 (ACE2), AT1/AT2 receptors, monocyte chemotactic protein-1 (MCP-1), transforming growth factor $\beta 1$ (TGF- $\beta 1$ ) and collagens was characterized to check the potential injurious mechanisms involved. Cardio toxicity likely-induced by cyclosporine was evaluated by accumulation of macrophages/myofibroblasts and interstitial/ perivascular fibrosis. Blood pressure was measured noninvasively to check whether dietary cyclosporine induces hypertension.

\section{Experimental}

\section{Materials and methods}

Animals and blood pressure measurement: Male and female C57BL/6 mice, 8-12 weeks of age (22-30g body weight) that were purchased from the Jackson laboratory (Bar Harbor, ME, USA) were kept in a 12 -hour light/dark cycle, $60 \%$ humidity and temperature- 
controlled room with standard rat chow and water ad libitum. These procedures were in compliance with "The Guide for the Care of Use of Laboratory Animals" published by the US National Institute of Health, 8th edition, 2011. The animal experimental procedures were approved by the Institutional Animal Care and Use Committee, Mercer University School of Medicine. The blood pressure was measured in conscious rats using a noninvasive blood pressure measuring system (AD Instruments NIBP controller, CO, USA). This system detects the signals through a transducer by the periodic occlusion of blood flow in the tail.

Experimental protocol and group: Five weeks of experimental period was selected for all experimental groups. Mice were randomly assigned into one of five groups: 1) Saline control (Sham, $n=6$ ): Saline ( $2 \mathrm{ml}$ ) were fed by gastric gavage daily; 2) Low dose of cyclosporine (CycL, $n=7)$ : cyclosporine ( 25 mg capsule, Apotex Corp. Florida, USA) was administered at a dose of $15 \mathrm{mg} / \mathrm{kg}$ /day by gastric gavage; 3) High dose of cyclosporine $(\mathrm{CycH}, \mathrm{n}=7)$ : cyclosporine was administered at a dose of $30 \mathrm{mg} / \mathrm{kg} /$ day by gastric gavage; 4) telmisartan+high dose of cyclosporine (Telmi, $\mathrm{n}=7$ ): telmisartan (Boehringer Ingelheim Pharmaceuticals, Inc., Ridgefield, CT, USA) at a dose of $10 \mathrm{mg} / \mathrm{kg} /$ day plus $30 \mathrm{mg} / \mathrm{kg} /$ day of cyclosporine were co-administered by gastric gavage; 5) curcumin+high dose of cyclosporine (Cur ): Curcumin (Cur, n=7): Curcumin (Sigma-Aldrich, CO, USA) at a dose of $150 \mathrm{mg}$ / $\mathrm{kg} /$ day plus $30 \mathrm{mg} / \mathrm{kg} /$ day of cyclosporine were co-administered by gastric gavage. The doses of cyclosporine selected were primarily based on previous studies showing deleterious effects of the drug on renal fibrosis and dysfunction ${ }^{4,7,11}$ At the end of 5 weeks of the experiment, the mice from different groups were euthanized and the hearts were removed.

\section{Western blot analysis of ACE2,ATI and AT2 receptors, MCP-I,TGF $\square$ I and collagens}

The freshly-frozen transmural cardiac tissue was homogenized in ice-cold lysis buffer. Total proteins $(60 \mu \mathrm{g})$ were extracted, fractionated by $10 \%$ SDS-polyacrylamide gel electrophoresis, and transferred to polyvinylidene difluoride membrane as described previously. ${ }^{9}$ The membrane was then incubated at $4^{\circ} \mathrm{C}$ overnight with the following antibodies: a rabbit anti-ACE2 polyclonal antibody (1:800), rabbit anti-AT1 (1:800) and AT2 (1:400) receptor polyclonal antibodies (Santa Cruz Biotech, Dallas, TX, USA), a rabbit antiMCP-1 polyclonal antibody and a mouse monoclonal anti-TGF $\beta 1$ antibody (1:1000, Abcam, Inc. MA, USA), and mouse anti-collagen type I and III monoclonal antibodies (1:5000, Sigma Chemical Co., MO, USA), respectively. Bound antibody was detected by horseradish peroxidase-conjugated anti-mouse IgG. The membrane was incubated with chemiluminescence detection reagents and exposed to an x-ray film. The images were then imported into the ImageJ (NIH, MA, USA) and quantified as a ratio based on the density of the target protein band divided by actin to correct differences during protein loading and/or transfer.

\section{Immunohistochemical staining of macrophages and myofibroblasts}

The paraffin-embedded tissue sections ( $4 \mu \mathrm{m}$ thick) were dewaxed in xylene, and rehydrated through graded alcohols as previously reported. ${ }^{9}$ Briefly, the sections were incubated overnight with primary antibodies including a mouse monoclonal antibody against macrophages (1:200, Millipore, CA, USA) and a monoclonal antibody against $\alpha$-smooth muscle actin (SMA, 1:800, Sigma Chemical Co., MO, USA), respectively. The slides were incubated with a biotinylated horse anti-rabbit IgG or an anti-mouse IgG (Vector Laboratories), stained using the $\mathrm{ABC}$-peroxidase kit or $\mathrm{ABC}-\mathrm{AP}$ (Alkaline phosphatase, Vector Laboratories, CA, USA) and visualized with 3,3'-diaminobenzidine tetrahydrochloride or alkaline phosphatase substrate kit (Sigma, MO, USA). Quality of immunohistochemistry assay was controlled by either elimination of the primary antibody or incubation of the tissue with a non-immune IgG. Sections were counterstained with hematoxylin, dehydrated and mounted by routine methods. The accumulation of macrophages and $\alpha$-SMA-expressing myofibroblasts among groups were compared using computedassisted morphometry (ImageJ, NIH). The final results were reported from the eight randomized high-powered fields.

\section{Masson's trichrome staining of collagen deposition}

The transversal myocardial blocks were fixed in phosphatebuffered formalin solution and dehydrated in graded series of alcohol, and embedded in paraffin as previously reported. ${ }^{9}$ Samples were sectioned to a thickness of $6 \mu \mathrm{m}$ using a Microtome (Leica RM2135, Meyer Instruments, TX, USA) and stained by Masson's trichrome producing collagen blue, nuclei black and muscle fiber red (Poly Scientific R\&D Corp, NY, USA). Ten randomized high-powered fields (HPF) per tissue section were evaluated to define the interstitial and perivascular fibrosis. All histological evaluation was performed by a single well-trained observer using a digital image analyzer (ImageJ, NIH, MA, USA).

\section{Statistical analysis}

Data were reported as the mean \pm standard error. A one-way ANOVA followed by Student-Newman-Keul's post hoc test was used to analyze group differences in the intensity of ACE2, AT1/ AT2 receptors, MCP-1, TGF $\beta 1$ and collagens, the populations of macrophages and myofibroblasts and change in blood pressure using Sigma Plot (Systat Software Inc., CA, and USA). Statistical significance was set at a value of $\mathrm{p}<0.05$.

\section{Results}

\section{Effect of dietary cyclosporine treatment on protein levels of ACE2,ATI and AT2 receptors}

The protein levels of ACE2, AT1 and AT2 receptors were analyzed using Western blot assay. As shown in Figure 1A, low or high dose of dietary cyclosporine significantly increased expression of ACE2 at week 5 relative to the sham control. Although co-administration of telmisartan or curcumin with cyclosporine also enhanced ACE2 relative to the sham control, there was no statistical difference when these values were compared with cyclosporine treatment alone. The AT1 receptor was detected in the sham condition (Figure 1B). However, low or high dose of cyclosporine treatment did not alter protein level of the AT1 receptor at week 5. Expression of the AT1 receptor was also not influenced by co-administration of telmisartan or curcumin with cyclosporine. As shown in Figure 1C, low or high dose of cyclosporine did not change protein level of the AT2 receptor relative to the sham control at week 5. Expression of the AT2 receptor was also not altered by co-administration of telmisartan or curcumin with cyclosporine over 5 weeks. 


\section{Effect of dietary cyclosporine treatment on protein levels of MCP-I,TGF $\square$ I, collagen I and collagen III}

The protein levels of MCP-1, TGF $\beta 1$, collagen I and collagen III were analyzed using Western blot assay. As shown in Figure 2, MCP1 TGF $\beta 1$, collagen I and collagen III were constitutively expressed in the sham control. At week 5, administration of low or high dose of dietary cyclosporine had no significant effect on protein levels of these parameters measured. Furthermore, these protein levels were also not altered by co-administration of telmisartan or curcumin with cyclosporine (Figure 2).

\section{Effect of dietary cyclosporine treatment on macrophage accumulation and myofibroblast proliferation}

Macrophage accumulation and myofibroblast proliferation were detected using immunohistochmical staining. As shown in Figure $3 \mathrm{~A}$, a few macrophages were detected in the myocardium in the sham group. Dietary high dose of cyclosporine had no significant effect on macrophage accumulation relative to the sham control at week 5 . Furthermore, co-administration of telmisartan or curcumin with high dose of cyclosporine for 5 weeks also did not affect the number of positively-stained macrophages relative to cyclosporine treatment alone. The proliferation of fibroblasts was identified as accumulation of the $\alpha$-SMA-expressing myofibroblasts. As shown in Figure 3B, a few of $\alpha$-SMA expressing myofibroblasts were only present in the vascular smooth muscle, but not in the myocardium in the sham group. Furthermore, dietary cyclosporine treatment did not produce a significant increase in the number of proliferated fibroblasts relative to the sham control. Furthermore, 5 weeks of co-administration of telmisartan or curcumin with cyclosporine also did not change the number of $\alpha$-SMA expressing myofibroblasts compared with cyclosporine treatment alone.

Effect of treatment with dietary cyclosporine on collagen deposition in the interstitial myocardium and perivascular region

Collagen deposition in the perivascular region and interstitial myocardium was further examined using Masson's trichrome staining. As shown in Figures 4A \& Figure 4B, no collagen deposition in the interstitial myocardium, the tunica intima and media of the microvessels was detected at the end of 5 weeks of the experiment in the sham group. In this normal condition, collagen positively-stained position was only preferentially partitioned into the adventitia (Figure $4 C)$. Dietary cyclosporine or co-administration of telmisartan or curcumin with cyclosporine for 5 weeks had no significant effect on collagen deposition (Figure 4). These data were consistent with lack of stimulating effect of cyclosporine on expression of collagen I and III as measured by Western blot assay.

\section{Effect of treatment with dietary cyclosporine on blood pressure}

Cyclosporine treatment has been associated with development of hypertension in kidney transplant recipients. ${ }^{1,2}$ As shown in Figure 5, the mean arterial pressure (MAP) was averaged by $56 \pm 8 \mathrm{mmHg}$ in the sham group. Low dose of cyclosporine treatment for 5 weeks had no significant effect on MAP. MAP in the high dose of cyclosporine group was averaged by $60 \pm 7 \mathrm{mmHg}$, which was $6.6 \pm 0.5 \%$ higher than that in the sham control, but it did not get a statistical difference.
There was a trend in reduction in blood pressure when telmisartan or curcumin was co-administered with high dose of cyclosporine, but, it did not get a statistical difference $(58 \pm 5 \mathrm{mmHg}$ in telmisartan group and $54 \pm 9 \mathrm{mmHg}$ in curcumin group vs. $60 \pm 7 \mathrm{mmHg}$ in cyclosporine group, all $p>0.05$, Figure 5), suggesting lack of stimulating effect of dietary cyclosporine treatment on blood pressure via Ang II activation and inflammatory responses in healthy animals.

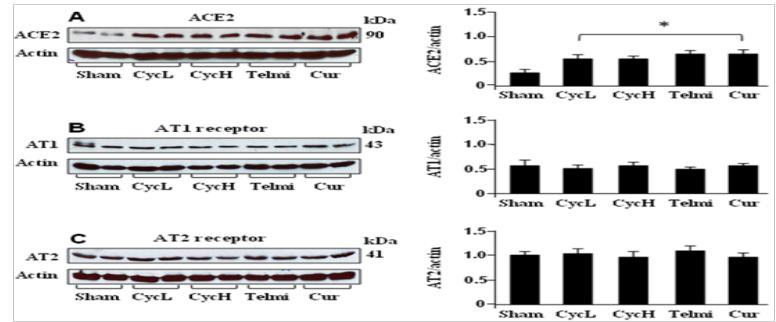

Figure I Expression of ACE2, ATI and AT2 receptor at the end of 5 weeks of dietary cyclosporine treatment. A, B and $C$ are representatives of tissue protein levels of ACE2,ATI and AT2 receptor by Western blot. All bands were normalized by actin as a loading control. Saline control (Sham); low dose of cyclosporine $(10 \mathrm{mg} / \mathrm{kg} /$ day, CycL); high dose of cyclosporine $(30 \mathrm{mg} / \mathrm{kg} /$ day, $\mathrm{CycH}$ ); telmisartan+high dose of cyclosporine (Telmi), curcumin+high dose of cyclosporine (Cur). Values are mean \pm SEM; $n=7$ for each group. ${ }^{*} p<0.05$ all group vs Sham.

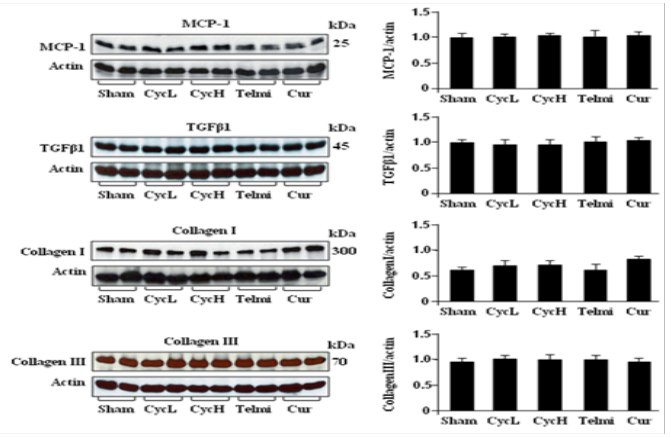

Figure 2 Expression of MCP-I,TGF $\square$ I, collagen I and collagen III at the end of 5 weeks of dietary cyclosporine treatment. The tissue protein levels of MCP-I,TGF $\square$ I, collagen I and collagen III were analyzed by Western blot. All bands were normalized by actin as a loading control. Values are mean \pm SEM; $n=7$ for each group.

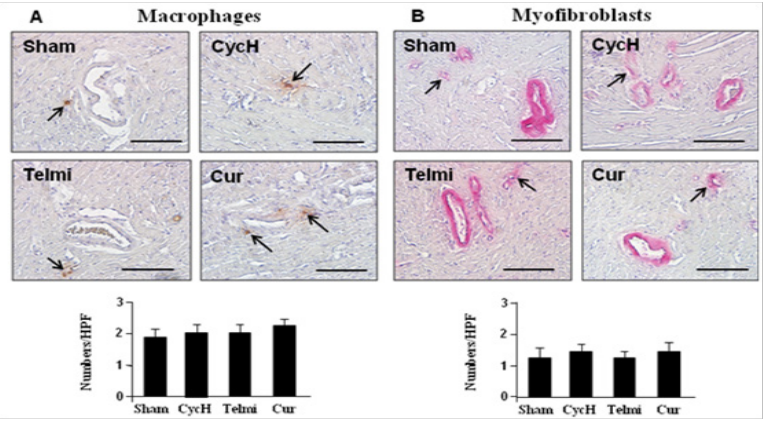

Figure 3 Macrophage accumulation and myofibroblast proliferation at the end of 5 weeks of dietary cyclosporine treatment. The macrophages and myofibroblasts were detected using immunohistochemical staining at each high powered field (HPF, magnification $\times 400$, scale bars $=200 \mu \mathrm{m})$. The myocardial tissue slides obtained from the heart after Ang II infusion were used as positive control for staining of macrophages and myofibroblasts. A few positively-stained macrophages and myofibroblasts (shown by arrows) were found among groups. Values are mean \pm SEM; $n=7$ for each group. 

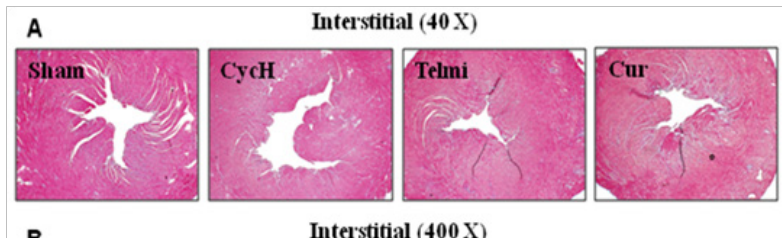

B
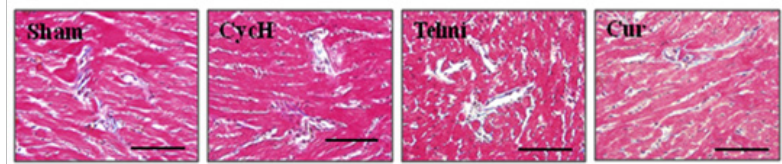

C

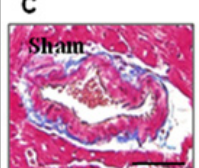

Perivascular $(400 \mathrm{X})$
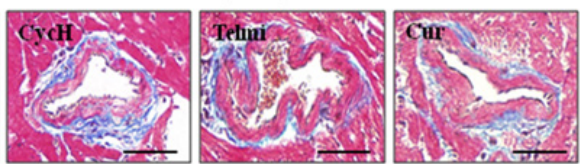

Figure 4 Representative photomicrographs of collagen deposition from the tissue sections and microvessels stained with Masson's trichrome staining. No newly-formed collagen was deposited in the interstitial myocardium ( $A$ and $B$ ) and perivascular region (C) at the end of 5 weeks of dietary cyclosporine treatment. Magnification in A is $\times 40$. Magnification and scale bars in B and C are $x 400$ and $100 \mu \mathrm{m}$, respectively. $\mathrm{n}=7$ for each group.

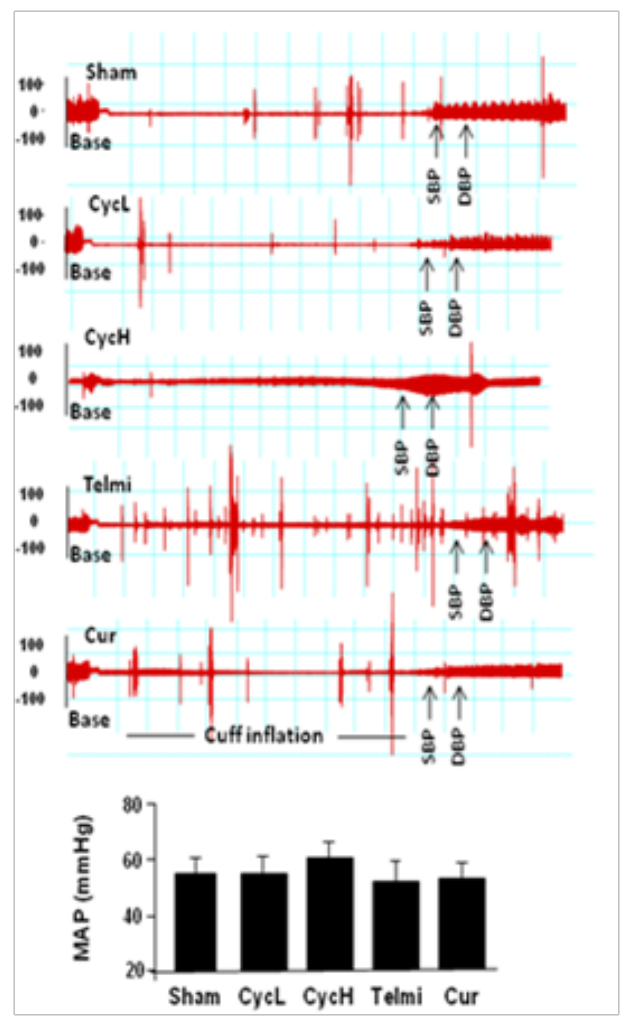

Figure 5 Change in blood pressure among groups at the end of 5 weeks of dietary cyclosporine treatment. The arrows indicate systolic blood pressure (SBP) and diastolic blood pressure (DBP). Difference in blood pressure among groups can be identified by a distance between cuff inflation from baseline (Base) and beginning of SBP tracing. Treatment with cyclosporine, telmisartan and curcumin did not significantly affect blood pressure as calculated from a mean arterial pressure (MAP). Values are mean $\pm S E M ; n=7$ for each group.

\section{Discussion}

Treatment with cyclosporine has been associated with chronic nephropathy and hypertension. It was unexpected that the present study does not show the results as initially hypothesized. We found that treatment with cyclosporine at the low or high dose for 5 weeks does not produce any significant deleterious effects on the heart, evidence by lack of significant macrophage accumulation, myofibroblast proliferation, collagen deposition, fibrosis and hypertension. Furthermore, cyclosporine did not alter protein expression of Ang II AT1/AT2 receptors, MCP-1, TGF $\beta 1$ and collagens, but significantly enhanced the expression of ACE2.

Cyclosporine has been used as one of the most potent immunosuppressive agents to prolong survival of transplanted organs. The primary concern to use cyclosporine in patient is druginduced impairment of renal function due to the dose and duration of cyclosporine administered. ${ }^{12}$ A previous retrospective study to evaluate the potential adverse effect of cyclosporine on kidney in patients found that cyclosporine treatment at a dose of $8 \mathrm{mg} / \mathrm{kg} /$ day for one year causes significant damage on kidney, defined as at least moderate focal interstitial fibrosis and large increase in the serum creatinine level. This chronic cyclosporine nephropathy can be minimized when the drug dose was selected in less than $5 \mathrm{mg} / \mathrm{kg} /$ day. ${ }^{13}$ In animal studies, it has also been shown that subcutaneous injection of cyclosporine at a dose of $30 \mathrm{mg} / \mathrm{kg} /$ day for 1 -month period of time significantly induces acute nephropathy and hypertension in rats and mice. ${ }^{7,8}$ In the present study, we chose the route of oral cyclosporine administration because of its clinical relevance in patient treatment to evaluate the potential adverse effect of cyclosporine on the heart. Data clearly showed that dietary cyclosporine in the dose range from 10 to $30 \mathrm{mg} / \mathrm{kg} /$ day does not produce obvious adverse effects on all the parameters measured. Although we did not measure the plasma concentration of cyclosporine, according to the pharmacokinetic report following oral administration in patients (drugs.com), absorption of cyclosporine is incomplete and $6 \%$ of the dose is excreted in urine. The time to peak blood cyclosporine concentrations ranged from 1.5 to 2 hours and the disposition of cyclosporine from blood is generally biphasic, with a terminal half-life of approximately 8.4 hours (range 5 to 18 hours). It has been proposed that mice are resistant to cyclosporine-induced renal injury due to the species specificity of rodents. ${ }^{4}$ Based on previous reports showing that one-month period of subcutaneous cyclosporine administration at the dosage range of 15 to $30 \mathrm{mg} / \mathrm{kg} /$ day in hypertensive mice causes significant change on renal function, ${ }^{4,5}$ we hypothesized that such a dose regimen of cyclosporine in this short period of time may also produce the adverse effects on the heart. Clearly, with these doses of cyclosporine, we were unable to demonstrate the any alterations in collagen protein expression/deposition and fibrosis. Different results obtained from the present study in the heart and previous studies in the kidney suggest that the kidney is more susceptible to cyclosporine-mediated effect when the drug is delivered subcutaneously. ${ }^{4,5}$ As a limitation of the present study, we did not determine the oral bioavailability of cyclosporine after garage administration. It has been previously reported that the oral bioavailability is associated with drug solubility and intestinal absorption. ${ }^{14,15}$ Data obtained from the rats have shown that cyclosporine can be detected in the blood at 2 hours after oral administration, and the bioavailability using intact cyclosporine is estimated by $40 \%$. Enhancement in solubility of cyclosporine with liposomes ${ }^{14}$ or in absorption of cyclosporine with endotoxin ${ }^{15}$ has 
shown an improvement in the oral bioavailability of cyclosporine. However, it remains to be determined whether using simulated doses of cyclosporine in patient could lead to myocardial fibrosis after a prolonged period of administration in larger hypertensive animals ${ }^{16}$

We have previously reported that infusion of Ang II in rat stimulates the expression of the AT1 receptor, down regulates the AT2 receptor and attenuates ACE2 activity, which are followed by macrophage/ myofibroblast-mediated activation of TGF $\beta 1 /$ collagen signaling. Furthermore, the inhibition of the AT1 receptor with the AT1 receptor antagonist or blockade of inflammation using an anti-inflammatory compound reduced myocardial fibrosis, primarily mediated by up regulating the AT2 receptor and ACE2 activity. ${ }^{9,10}$ In previous animal studies, the side effects of cyclosporine on kidney have been associated with activation of Ang II AT1 receptor and induction of inflammatory mediators. Concurrent administration of the AT1 receptor antagonist or a superoxide dismutase mimetic prevented cyclosporine-induced nephropathy and hypertension ${ }^{5,6}$ Furthermore, selective stimulation of the AT2 receptor also attenuated cyclosporine-mediated renal fibrosis. ${ }^{11,16}$ In the present study, we detected expression of the AT1 and AT2 receptors to reflect the Ang II activation as we initially proposed, but, we did not find that these receptors are stimulated by cyclosporine. We have previously demonstrated that ACE2 is present in the vascular endothelium, smooth muscle cells of coronary vessels and cardio myocytes, and the reduced expression of ACE2 has been associated with development of hypertension, inflammation and tissue fibrosis. ${ }^{9} 10$ In the present experimental setting, we found that the expression of ACE2 is significantly up regulated following dietary cyclosporine alone. No further increase in expression of ACE2 was detected when cyclosporine was co-administered with telimisartan or curcumin, suggesting that cyclosporine has direct effect on activation of ACE2. It is worth to further explore the mechanisms of action involved cyclosporine-activated ACE2 and the role of ACE2 in cyclosporine-mediated injury in kidney.

We have reported that MCP-1 plays a major role in mediating macrophage migration after infusion of Ang II or myocardial infarction. ${ }^{9}$ To understand a signaling pathway, we have also demonstrated that TGF $\beta 1$ released from activated macrophages is responsible for myofibroblast proliferation and collagen production. In the present study, in coincidence with unchanged protein expression of MCP-1 after treatment with cyclosporine alone or co-administration of cyclosporine with telmisartan or curcumin, the number of positively-stained macrophages in the interstitial myocardium was remained unchanged as compared with those in the sham control. Furthermore, no significant myofibroblast proliferation and collagen deposition were identified. These data suggest that treatment of dietary cyclosporine for 5 weeks at the dose regimen as selected in the present study does not stimulate macrophage/myofibroblast/TGF $\beta 1$-mediated collagen production. Accordingly, cyclosporine also did not produce tissue fibrosis as identified by absence of interstitial/perivascular fibrosis via Masson's trichrome staining. Our results were consistent with previous study showing that cyclosporine has no direct effect on production and deposition of collagen released from the cultured cardiac fibroblasts as compared with untreated cells. ${ }^{17}$ Although it has been suggested that treatment with cyclosporine may associate with frequency of cardiac hypertrophy and interstitial fibrosis in heart transplant recipients, ${ }^{18}$ our data and others suggested that other mediators, which may be released during cardiac allograft, but not with cyclosporine, are responsible for the tissue fibrosis. ${ }^{18,19}$
Increase in blood pressure has been associated with chronic cyclosporine treatment in animals and patients after kidney or heart transplantation. ${ }^{18-20}$ The present study was performed on normal animals. Although we found a minor increase in blood pressure, it did not reach a significant level. These data also suggest that change in blood pressure with cyclosporine is potentially induced through transplanted organs. For example, different mechanisms have been proposed in the induction of cyclosporine-induced hypertension in kidney-transplant recipients, including reduction in blood flow to kidney, alteration in vasoactive mediators such as renin, endothelin-1 and promotion of endothelial dysfunction. ${ }^{21}$ On the other hand, augmented sympathetic activation is also thought to be one possible explanation for the greater hypertensive effect of cyclosporine in heart-transplant recipients than in individuals treated with other interventions. In these recipients, the sympathoexcitatory effect of cyclosporine may be accentuated because the transplanted heart interrupts the neutral connection to the central nervous system and removes baroreceptor restraint on sympathetic outflow. ${ }^{20,}{ }^{22} \mathrm{We}$ initially hypothesized that cyclosporine treatment increases blood pressure and this change may be mediated by activation of Ang II and induction of inflammation. Although we found that there is a trend in reduction in blood pressure when cyclosporine was co-administered with telmisartan and curcumin relative to cyclosporine alone, it did not achieve a statistical significant level. These data further support that cyclosporine derived hypertension is associated with the transplanted organs, but not in healthy individuals.

Instead of the data showing cyclosporine-induced hypertension and tissue injury in transplanted organs, cardio protection with cyclosporine has also attracted quite some attention. Clinical studies have recently shown that intravenous bolus injection of cyclosporine at the onset of reperfusion significantly reduces infarct size as estimated by a reduction in release of cardiac tropornin I after percutaneous coronary intervention ${ }^{23}$ or the aortic cross-clamping during aortic valve surgery. ${ }^{24}$ However, later on in the follow-up observations, these investigators have also reported that single injection of cyclosporine at reperfusion dose not result in better clinical outcomes and prevent adverse ventricular remodeling than those with placebo treated patients. ${ }^{25}$ Because the half-life of cyclosporine is about a few hours from administration, it may be difficult to achieve a high degree of long-term protective effect on cardiac remodeling with a single bolus injection after myocardial infarction. In this regard, a previous study has demonstrated that pressure overload induced left ventricular hypertrophy via aortic banding is significantly attenuated by cyclosporine at a dose of $25 \mathrm{mg} / \mathrm{kg} / /$ day for 22 days, as evidenced by reduced ventricular wall stress. ${ }^{26}$ Our current data, which are lack of any deleterious effects on the heart and blood pressure with cyclosporine, were consistent with a previous report in patients showing that cyclosporine treatment in heart transplant recipients does not produces significant myocardial collagen volume fractions, vascularity and fibrosis. ${ }^{27}$ Based on these findings, we propose that there may be a better option to improve cardiac remodeling and outcomes in patient if cyclosporine is orally administered daily after myocardial infarction and during the progression of heart failure..$^{25,28}$

\section{Conclusion}

In summary, this study demonstrates that dietary cyclosporine alone or co-administration with either telmisartan or curcumin has no direct adverse effects on myocardial morphology and blood pressure in normal condition. These data suggest that chronic nephropathy, 
hypertension and tissue fibrosis identified in organ recipients may not be directly induced by cyclosporine. In these conditions, pathogenesis such as augmented Ang II activity and inflammation evoked by post-transplant lesions may increase organ susceptibility to injury seen during cyclosporine treatment. Our findings may support the future study to further differentiate direct effect of cyclosporine on transplanted organs regarding therapeutic regimen such as drug dosage, administration route and treatment duration. Furthermore, our data may also provide experimental evidence to consider the therapeutic window of cyclosporine as an alternative adjunctive therapy with commonly-used drugs such as ACE inhibitors, Ang II AT1 receptor blocker or anti-inflammatory agent, and fully protect the heart against the tissue injury resulting from the different cardiovascular diseases and heart failure.

\section{Acknowledgements}

This study was supported in part by the Mercer University School of Medicine, the Rubye Smith Trust Funds, the Medcen Community Health Foundation, Georgia and the National Natural Science Foundation of China (81470436).

\section{Conflict of interest}

The author declares no conflict of interest.

\section{References}

1. Guada M, Beloqui A, Kumar MN, et al. Reformulating cyclosporine A (CsA): More than just a life cycle management strategy. J Control Release. 2016;225:269-282.

2. Hami M, Mojahedi MJ, Naghibi M, et al. Cyclosporine trough levels and its side effects in kidney transplant recipients. Iran J Kidney Dis. 2010;4(2):153-157.

3. Karam S, Wali RK. Current state of immunosuppression: past, present, and future. Eukaryot Gene Expr. 2015;25(2):113-134.

4. Yoon HE, Ghee JY, Piao S, et al. Angiotensin II blockade up regulates the expression of Klotho, the anti-ageing gene, in an experimental model of chronic cyclosporine nephropathy. Nephrol Dial Transplant. 2011;26(3):800-813.

5. Lassila M, Finckenberg P, Pere AK, et al. Comparison of enalapril and valsartan in cyclosporine A-induced hypertension and nephrotoxicity in spontaneously hypertensive rats on high-sodium diet. Br J Pharmacol. 2000;130(6):1339-1347.

6. Ebru U, Burak U, Yusuf S, et al. Cardioprotective effects of Nigella sativa oil on cyclosporine A-induced cardiotoxicity in rats. Basic Clin Pharmacol Toxicol. 2008;103(6):574-580.

7. Nishiyama A, Kobori H, Fukui T, et al. Role of angiotensin II and reactive oxygen species in cyclosporine A-dependent hypertension. Hypertension. 2003;42(6):754-760.

8. Sereno J, Rodrigues-Santos $\mathrm{P}$, Vala $\mathrm{H}$, et al. Transition from cyclosporineinduced renal dysfunction to nephrotoxicity in an in vivo rat model. Int $J$ Mol Sci. 2014;15(5):8979-8997.

9. Bai F, Pang XF, Zhang LH, et al. Angiotensin II AT1 receptor alters ACE2 activity, eNOS expression and CD44-hyaluronan interaction in rats with hypertension and myocardial fibrosis. Life Sci. 2016;153:141-152.

10. Pang XF, Zhang LH, Bai F, et al. Attenuation of myocardial fibrosis with curcumin is mediated by modulating expression of angiotensin II AT1/AT2 receptors and ACE2 in rats. Drug Des Devel Ther. 2015;9:6043-6054.
11. Castoldi G, di Gioia CR, Carletti R, et al. Angiotensin Type-2 (AT-2)Receptor activation reduces renal fibrosis in cyclosporine nephropathy: Evidence for blood-pressure independent effect. Biosci Rep. 2016;36:e00403.

12. Colombo MD, Cassano N, Bellia G, et al. Cyclosporine regimens in plaque psoriasis: an overview with special emphasis on dose, duration, and old and new treatment approaches. Scientific World J. 2013;25:e805705.

13. Isnard Bagnis $\mathrm{C}$, Tezenas du Montcel $\mathrm{S}$, Beaufils $\mathrm{H}$, et al. Long-term renal effects of low-dose cyclosporine in uveitis-treated patients: follow-up study. J Am Soc Nephrol. 2002;13(12):2962-2968.

14. Guan P, Lu Y, Qi J, et al. Enhanced oral bioavailability of cyclosporine A by liposomes containing a bile salt. Int J Nanomedicine. 2011;6:965-974.

15. Prasad V, Jain V, Mishra PR. Bioavailability enhancement of cyclosporine in rats pretreated with endotoxin. $J$ Pharmacology \&Toxicology. 2006;1(1):54-63.

16. Yamauchi H, Kobayashi E, Sugimoto K, et al. Time-dependent cyclosporine A-induced nephrotoxicity in rats. Clin Exp Pharmacol Physiol. 1998;25(6):435-440.

17. Eleftheriades EG, Ferguson AG, Samarel AM. Cyclosporine A has no direct effect on collagen metabolism by cardiac fibroblasts in vitro. Circulation. 1993;87(4):1368-1377.

18. Gramley F, Lorenzen J, Pezzella F, et al. Hypoxia and myocardial remodeling in human cardiac allografts: a time-course study. J Heart Lung Transplant. 2009;28(11):1119-1126.

19. Hunt SA, Haddad F. The changing face of heart transplantation. J Am Coll Cardiol. 2008;52(8):587-598.

20. Morgan BJ, Lyson T, Scherrer U, et al. Cyclosporine causes sympathetically mediated elevations in arterial pressure in rats. Hypertension. 1991;18(4):458-466.

21. Kang DH, Kim YG, Andoh TF, et al. Post-cyclosporine-mediated hypertension and nephropathy: amelioration by vascular endothelial growth factor. Am J Physiol Renal Physiol. 2001;280(4):F727-736.

22. Scherrer U, Vissing SF, Morgan BJ, et al. Cyclosporine-induced sympathetic activation and hypertension after heart transplantation. $N$ Engl J Med. 1990;323(11):693-699.

23. Cung TT, Morel O, Cayla G, et al. Cyclosporine before PCI in Patients with Acute Myocardial Infarction. N Engl J Med. 2015;373(11):1021-1031.

24. Chiari P, Angoulvant D, Mewton N, et al. Cyclosporine protects the heart during aortic valve surgery. Anesthesiology. 2014;121(2):232-238.

25. Mewton N, Cung TT, Morel O, et al. Rationale and design of the cyclosporine to improve Clinical outcome in ST-elevation myocardial infarction patients (the CIRCUS trial). Am Heart J. 2015;169(6):758-766.

26. Meguro T, Hong C, Asai K, et al. Cyclosporine attenuates pressure-overload hypertrophy in mice while enhancing susceptibility to decompensation and heart failure. Circ Res. 1999;84(6):735-740.

27. Rowan RA, Billingham ME. Pathologic changes in the long-term transplanted heart: a morphometric study of myocardial hypertrophy, vascularity, and fibrosis. Hum Pathol. 1990;21(7):767-772.

28. Nighoghossian N, Ovize M, Mewton N, et al. Cyclosporine A, a potential therapy of ischemic reperfusion injury. A common history for heart and brain. Cerebrovasc Dis. 2016;42(5-6):309-318. 